\title{
Design and Experiment on the Compact, Portable Pulse Forming Line
}

\author{
L. Xia*, J. Shi, D. Chen, H. Zhang, L. Zhang and J. Deng \\ Institute of Fluid Physics, Chinese Academy of Engineering Physics \\ Mianyang 621900, China
}

\begin{abstract}
Some research has been done on making the compact, portable pulse forming line. A kind of ceramic and a kind of newly developed composite polymer are both used as the dielectric of the planar pulse forming line. The ceramic's dielectric constant ranges from 200 to 600 and the composite polymer's dielectric constant ranges from 100 to 200 . It is easier to manufacture the large composite polymer dielectric bulks than to manufacture the ceramic bulks. In the experiments, using the large composite polymer bulk as the dielectric, the planar Blumlein line generated the pulse up to $100 \mathrm{kV}$ with duration $90 \mathrm{~ns}$. Using the ceramic bulk as the dielectric, the planar line generated the voltage pulse up to $60 \mathrm{kV}$ with duration $92 \mathrm{~ns}$.
\end{abstract}

PACS numbers: $84.70 .+\mathrm{p}, 77.90 .+\mathrm{k}$

\section{Introduction}

Modern applications require compact, portable pulsed power systems [1]. The American government once supported a program titled MURI to study compact, portable pulsed power system [2]. Capacitive storage-based pulse forming lines are typically used as HPM source drivers and compact pulsed laser source drivers [3]. The requirements include maintaining constant impedance at $100 \mathrm{kV}-1 \mathrm{MV}$ voltages for time scales on the order of $100 \mathrm{~ns}$. Traditional materials used in pulse forming lines are primarily plastics or oil whose dielectric constants range from 2 to 10 . If one can use a material whose dielectric constant is larger than that of the plastic and the oil, then the pulse forming line's length can be greatly reduced for a given pulse duration. Using water as the dielectric, the pulse forming line's length is only about one of five of the line's length using oil as the dielectric. However, it is not easy to maintain water's resistivity high and the line is not enough compact and portable either. To get more compact, portable pulsed power systems, the challenge lies in finding a material whose dielectric constant is larger than that of water and is also easy to maintain.

In this paper, we introduce the work that we have made to pursue the compact, portable pulse forming line using ceramic and a newly developed composite polymer as the energy storage dielectric.

\section{The design of the pulse forming lines}

\subsection{Ceramic as the dielectric}

To make the pulse forming line more portable and compact, it is easy to choose ceramic as the dielectric. Ceramic commonly has large dielectric constant (a few tens

* corresponding author; e-mail: lsh_xia@yahoo.com.cn to a few thousands) and high bulk breakdown strength (more than $300 \mathrm{kV} / \mathrm{cm}$ ) [4].

Although ceramic is an ideal dielectric for compact and portable transmission line, it is difficult to manufacture the large ceramic bulks. It took a few years to manufacture the large ceramic bulks. It is $\mathrm{SrTiO}_{3}$ based ceramic and the bulks are $80 \mathrm{~cm} \times 10 \mathrm{~cm} \times 6 \mathrm{~cm}$. The dielectric constant $\varepsilon_{\mathrm{r}}$ is 300 and quite steady from $100 \mathrm{kHz}$ to $50 \mathrm{MHz}$ and the relative permeability $\mu_{\mathrm{r}}$ is 1 . In Table the basic parameters of ceramic based on $\mathrm{SrTiO}_{3}$ are given. Because of the defects of the ceramic bulks, breakdown strength (BS) is much lower than expected. At the same time, surface flashover strength (SFS) is much lower than breakdown strength.

TABLE

Electric parameters of the ceramic used as the dielectric.

\begin{tabular}{c|c|c}
\hline \hline Parameter & Expected & Tested \\
\hline $\mathrm{BS}[\mathrm{kV} / \mathrm{cm}]$ & 300 & 100 \\
$\mathrm{SFS}[\mathrm{kV} / \mathrm{cm}]$ & 40 & 35 \\
DC resistivity $[\mathrm{M} \Omega \mathrm{cm}]$ & 500 & $>200$
\end{tabular}

Figure 1 shows the pulse forming line, which includes a ceramic bulk and two copper electrodes. The copper electrodes were stuck to the ceramic bulk with conductive glue. According to Eqs. (1) and (2), one can obtain that the impedance of the planar pulse forming line is $6.7 \Omega$ and the pulse duration is $92 \mathrm{~ns}$. In Eq. (1), $Z_{0}$ is the impedance of the line, $w$ is the width of the electrodes, and $d$ is the distance between the two electrodes. In Eq. (2), $l$ is the length of the bulk and $\tau_{0}$ is the pulse duration

$$
Z_{0}=\frac{377}{\sqrt{\varepsilon_{\mathrm{r}}}} \frac{d}{W+d},
$$




$$
\tau_{0}=\frac{2 \sqrt{\varepsilon_{\mathrm{r}} \mu_{\mathrm{r}}}}{c} l .
$$

Figure 2 is the voltage waveform generated by the pulse forming line shown in Fig. 1. Figure 2a is the result under a few kilovolts. The switch is a self breakdown air spark gap. Because the spark gap is very small, the gap inductance is small, too. Therefore the rise-time of the waveform is less than $15 \mathrm{~ns}$. The full width of the half maximum (FWHM) of the waveform is measured as $92 \mathrm{ns,}$ which is very close to the result calculated by Eq. (2). Figure $2 \mathrm{~b}$ is the result under a few tens kilovolts. A slow high voltage pulse generated by a Marx generator was used to charge the pulse forming line and the maximal amplitude of the charge pulse is about $120 \mathrm{kV}$. Because there is about $10 \mathrm{kA}$ electric current passing through the load, the traditional resistance is not suitable. A kind of solution is used as the load and the switch is a self breakdown oil gap. To sustain more than one hundred kilovolts, the oil gap was about $8 \mathrm{~mm}$. So the gap inductance is much larger and this made the rise-time of the waveform equal to about $30 \mathrm{~ns}$.

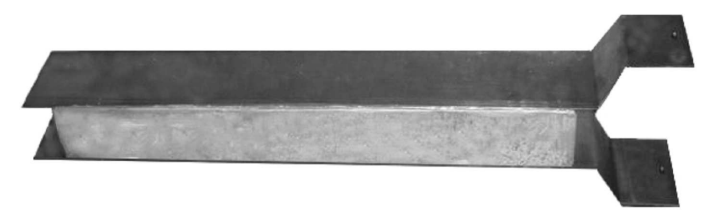

Fig. 1. Photo of the pulse forming line.

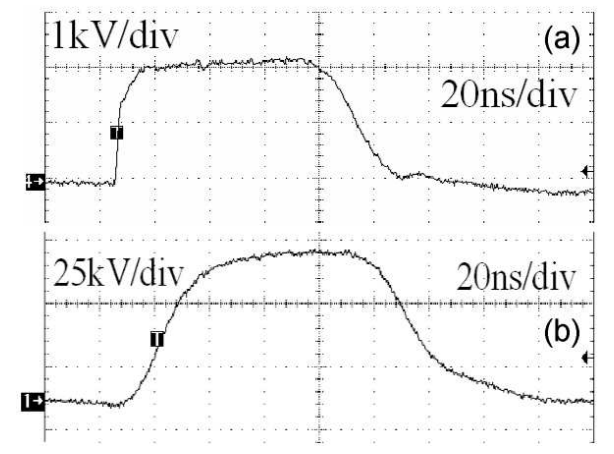

Fig. 2. Voltage waveforms generated by the pulse forming line: (a) waveform under low charging voltage, (b) waveform under high charging voltage.

\subsection{Composite polymer as the dielectric}

Another kind of compact, portable pulse forming line also was developed, which used a newly developed composite polymer as the dielectric. The dielectric constant of the composite polymer can range from 100 to 200 and keeps steady from $100 \mathrm{kHz}$ to $1000 \mathrm{MHz}$. Although the composite polymer bulk breakdown strength is much lower than that of the ceramic and is only about $100 \mathrm{kV} / \mathrm{cm}$, it is easy to manufacture large composite polymer bulks by using high pressure-heat amalgamation process. The composite polymer bulks based planar Blumlein line was made. Each bulk is $100 \mathrm{~cm} \times 20 \mathrm{~cm} \times 4 \mathrm{~cm}$. The line can generate a voltage pulse with the duration of 90 ns. The line's output impedance is $10 \Omega$ and the expected charging voltage can be up to $200 \mathrm{kV}$. Figure 3 shows the voltage waveform on the load. A compressed gas gap is used to short the line. However, the switch can only sustain voltage not more than $100 \mathrm{kV}$.

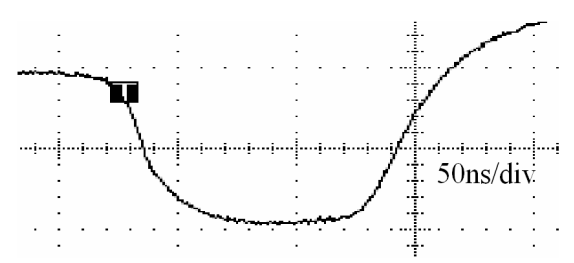

Fig. 3. Voltage waveform generated by the newly tested Blumlein line.

\section{Conclusion}

The high voltage pulse forming lines both using the ceramic and the composite polymer as the dielectric are made. The line using ceramic as the dielectric is more compact and needs no maintenance. At the same time, the ceramic dielectric bulk stores more energy than water in the same volume. The design and test results indicate that the kind of line based on $\mathrm{SrTiO}_{3}$ ceramic worked well. With suitable high voltage and low inductance switch, the pulse forming line has the ability to generate a voltage pulse more than $300 \mathrm{kV}$. The line which uses the large composite polymer bulks as the dielectric can generate pulsed voltage up to $200 \mathrm{kV}$ with duration 90 ns. Although the composite polymer bulk's breakdown strength is much lower than that of the ceramic, we can make much larger dielectric bulk with the composite polymer. Based on the work introduced, it is possible to make the compact, portable pulse forming line which can generate voltage pulse up to million volts (MV) with duration about 100 ns.

\section{References}

[1] R. Liu, P. Zheng, D. Xie, IEEE Trans. Magn. 43, 355 (2007).

[2] E. Schamiloglu, K. Schoenbach, R. Vidmar, in: Proc. SPIE, Intense Microwave Pulses IX, Orlando, FL, 2002, p. 1.

[3] E. Spahn, G. Buderer, W. Wenning, IEEE Trans. Magn. 35, 378 (1999).

[4] L.S. Xia, H. Zhang, J.S. Shi, J.J. Deng, L.W. Zhang, Rev. Sci. Instrum., to be published. 